\title{
Immunohistochemical Examination of Novel Rat Monoclonal Antibodies against Mouse and Human Podoplanin
}

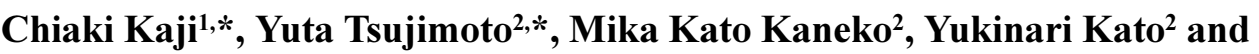 \\ Yoshihiko Sawa ${ }^{1}$ \\ ${ }^{1}$ Department of Morphological Biology, Fukuoka Dental College, 2-15-1 Tamura, Sawara-ku, Fukuoka 814-0193, Japan and \\ ${ }^{2}$ Molecular Tumor Marker Research Team, Yamagata University Global COE Program, Yamagata University Faculty of \\ Medicine, 2-2-2 Iida-nishi, Yamagata 990-9585, Japan
}

Received February 24, 2012; accepted June 14, 2012; published online July 25, 2012

\begin{abstract}
This study aims to develop new monoclonal antibodies (mAbs) against mouse and human podoplanin. Rats were immunized with synthetic peptides, corresponding to amino acids 3851 of mouse podoplanin or human podoplanin which is $100 \%$ homologous to the same site of monkey podoplanin; anti-mouse podoplanin mAb PMab-1 $\left(\operatorname{lgG}_{2 a}\right)$ and anti-human $\mathrm{mAb}$ $\mathrm{NZ}-1.2\left(\mathrm{IgG}_{2 a}\right)$ were established. In immunocytochemistry, the mouse melanoma B16-F10 and mouse podoplanin (mPDPN)-expressed $\mathrm{CHO}$ transfectant were stained by PMab-1; human lymphatic endothelial cells (LEC) and human podoplanin (hPDPN)-expressed squamous cell carcinoma HSC3 transfectant, were stained by NZ-1.2. Western-blot analysis detected an about $40-\mathrm{kDa}$ protein in CHO-mPDPN and B16-F10 by PMab-1, and in HSC3hPDPN and LEC by NZ-1.2. In frozen sections, PMab-1 reacted with mouse kidney, pulmonary alveoli, pulmonary pleura, and salivary gland myoepithelial cells while NZ-1.2 reacted to the human salivary gland myoepithelial cells. The immunostaining of paraffin-embedded sections also showed the reaction of PMab-1 or NZ-1.2 to the mouse or monkey kidney glomerulus, pulmonary alveoli, and lung lymphatic vessels. These results indicate that the two novel rat $\mathrm{mAbs}$ to the mouse and human/monkey podoplanin are useful for Western-blot and immunostaining of somatic tissues on paraffin-embedded sections as well as frozen sections.
\end{abstract}

Key words: podoplanin, monoclonal antibody, PMab-1, NZ-1.2

\section{Introduction}

Podoplanin, also termed Aggrus/gp36/E11 antigen, is a $38-\mathrm{kDa}$ (mouse) or $36-\mathrm{kDa}$ (human) mucin-type glycoprotein with a high content of sialic acid [7, 10]. Podoplanin was first identified as an antigen of the podocyte, the

\footnotetext{
*These authors contributed equally to this work.

Correspondence to: Yoshihiko Sawa, Department of Morphological Biology, Fukuoka Dental College, 2-15-1 Tamura, Sawara-ku, Fukuoka 814-0193, Japan. E-mail: ysawa@college.fdcnet.ac.jp

Yukinari Kato, Molecular Tumor Marker Research Team, Yamagata University Global COE Program, Yamagata University, Faculty of Medicine, 2-2-2 Iida-nishi, Yamagata 990-9585, Japan.

E-mail: yukinari-k@bea.hi-ho.ne.jp
}

kidney glomerular epithelial cell [1], and is also known as a marker of lymphatic endothelium and alveolar epithelium $[22,27,35]$. There has also been reports that podoplanin is expressed in osteocytes and osteoblasts [33], mesothelial cells [14], epidermal basal layer cells [27], tooth germ epithelial cells, salivary gland myoepithelium [4-6, 20], thymus type I epithelial cells, prostate myofibroblasts, follicular dendritic cells, and immature cells such as fetal germ cells or developing Sertoli cells [17, 22, 27, 35, 36]. The podoplanin promoter region lacks the TATA and CAT box, but includes a $\mathrm{CpG}$ island with putative Sp1 and AP-4 sites [3]. Such promoters are usually present at the 5'-flanking region of housekeeping genes, and $\mathrm{CpG}$ hypermethylation has been linked with their transcriptional repression. There is, however, $\mathrm{CpG}$ methylation-dependent podoplanin transcription enhancement, which is probably based on chroma- 
tin structure modifications; therefore, the $\mathrm{CpG}$ methylation may induce the specific expression in different kinds of somatic cells [3, 34].

The C-type lectin-like receptor 2 (CLEC-2) is the specific receptor for podoplanin, which mediates the platelet aggregation without blood coagulation factors and plasma components [12, 30]. Podoplanin consists of an extracellular domain rich in serine and threonine residues, and with a transmembrane domain, and a cytoplasmic tail for protein kinase $\mathrm{C}$ and CAMP phosphorylation [8]. Podoplanin has a platelet aggregation-stimulating (PLAG) domain, of which only the Thr52 is $O$-glycosylated with disialyl-core 1 (NeuAc- $\alpha 2-3$ Gal $\beta 1-3$ (NeuAc $\alpha 2-6)$ GalNAc $\alpha 1-$ $O$-Thr) is in an extracellular domain [9]. The binding of podoplanin to CLEC-2 is dependent on the sialic acid of $O$-glycan in the PLAG domain by the lectin activity, and mediates the platelet aggregation [12]. Three tandem repeats of the PLAG domain are conserved in podoplanin homologues of the rat, hamster, dog, bovine, human, and mouse [8]. The podoplanin homologues conserve the EDxxVTPG segment in the extracellular domains, which are critical for the platelet aggregation-inducing activities.

To date, several anti-mouse/human podoplanin antibodies have been established. The D2-40 antibody which recognizes the oncofetal antigen $\mathrm{M} 2 \mathrm{~A}$ of human podoplanin, and is useful in immunohistochemistry [2, 23]. Another anti-human podoplanin mAb (Clone AB3, AngioBio) possesses the very same characteristics as D2-40 [21]. The NZ1 antibody against the human PLAG domain suppresses both podoplanin-CELC-2 interaction and podoplanininduced hematogenous pulmonary metastasis $[11,12,21]$. The 8F11 monoclonal antibody against mouse podoplanin inhibits platelet aggregation and lung metastasis of mouse colon carcinomas [29, 31]; however, 8F11 has not been reported to be useful in immunohistochemistry. Podoplaninexpressing tumor cells may survive by aggregating platelets around the cells [12, 16], and podoplanin expression is associated with a poor prognosis of some kinds of tumors, and therefore podoplanin could be valuable as an anticancer target [13, 18, 28, 37]. Especially, the development of antibodies, which recognize the PLAG domain strongly, may contribute to anti-metastasis therapies against podoplanin-positive tumors. A number of anti-mouse/human podoplanin antibodies have been reported, however novel anti-podoplanin antibodies are still required to examine the precise distribution of podoplanin in somatic tissue. This study aims to develop new antibodies for the PLAG domain of mouse and human podoplanin, and investigates the specificity for podoplanin of new antibodies in the mouse, monkey, and human tissue.

\section{Materials and Methods}

\section{Subjects and cultured cells}

Human tissue of the sublingual glands of five individuals, 60-80 years of age, was obtained immediately following the body donation to Fukuoka Dental College for education and research of oral medicine. All procedures were performed with appropriate informed consent according to the protocol of the human gift registry programs of Fukuoka Dental College. The study used C57BL/6N mice (CLEA Japan, Inc., Tokyo, Japan) and Sprague-Dawley (SD) rats (CLEA) in the immunohistochemical examination and in the development of rat antibodies, respectively. The mouse myeloma P3U1 cells (CRL-1597) and Chinese hamster ovary (CHO) K1 cells (CRL-2243) were obtained from the American Type Culture Collection (ATCC, Manassas, VA). The human tongue squamous cell carcinoma cell line (HSC3) was obtained from the Japanese Collection of Research Bioresources Cell Bank (Osaka, Japan). The P3U1 and CHO cells or HSC3 were cultured in RPMI 1640 or Dulbecco's Modified Eagle Medium (DMEM, Wako Pure Chemical Industries Ltd., Osaka, Japan), respectively, including $2 \mathrm{mM}$ L-glutamine (Wako) supplemented with $10 \%$ heat-inactivated fetal bovine serum (FBS; Life Technologies Japan Ltd., Tokyo, Japan) at $37^{\circ} \mathrm{C}$ in a humidified atmosphere of $5 \% \mathrm{CO}_{2}$ and $95 \%$ air. The neonatal human dermal lymphatic microvascular endothelial cells (LEC: HMVEC-dLy, CC-2812, Lonza, Walkersville, MD) and mouse melanoma B16-F10 cells (CRL-6475, ATCC), which are known to express intrinsic podoplanin [32], were also used as a positive control for intrinsic podoplanin. The LEC and B16-F10 were cultured in endothelial cell medium EGM-2MV supplemented with $5 \%$ FBS (Lonza) or DMEM (Wako), respectively.

\section{Stable transfection}

The CHO cells were transfected with appropriate amounts of plasmid: pcDNA3-mouse podoplanin-DYKDDDDK (FLAG) [10] using LipofectAMINE 2000 reagent (Life Technologies Japan Ltd.) according to the manufacturer's instructions. The HSC3 cells were transfected with a plasmid: pcDNA3-human podoplanin-DYKDDDDK (FLAG) [10] by electroporation using CUY21Pro-Vitro (NepaGene Co., Ltd., Ichikawa, Japan) according to the original protocol. Briefly, the confluent monolayer of HSC3 cells in 6-well plates were trypsinized, harvested, and suspended in the Opti-MEM I (Life Technologies Japan Ltd.). A $10-\mu 1$ volume of the RNase and DNase freedeionized distilled water containing $2 \mu \mathrm{g}$ of pcDNA3human podoplanin was added into a $90-\mu \mathrm{l}$ volume of the HSC3 cell suspension $\left(1.0 \times 10^{6}\right)$ in the electroporation cuvettes (NepaGene). Electroporation was repeated 10 times: $125 \mathrm{~V}$ for $10 \mathrm{~ms}$, offtime for $50 \mathrm{~ms}, 20 \mathrm{~V}$ for $50 \mathrm{~ms}$, and off time for $50 \mathrm{~ms}$. After the electroporation, the cell suspension was immediately disseminated in DMEM with FBS, and the stable transfectants of CHO-mouse podoplanin (CHO-mPDPN) and HSC3-human podoplanin (HSC3-hPDPN) were selected by cultivating in a medium containing $0.5 \mathrm{mg} / \mathrm{ml}$ of Geneticin (Wako Pure Chemical Industries Ltd.). Mouse and human podoplanin genes in pcDNA3 were confirmed by sequence analysis (Macrogen Japan, Tokyo, Japan). 


\section{production of anti-mouse podoplanin and anti-human podoplanin monoclonal antibodies}

The SD rats were immunized by neck subcutaneous injections of the synthetic peptide GDGMVPPGIEDKIT (mpp3851) or EGGVAMPGAEDDVV (hpp3851), corresponding to amino acids 38-51 of mouse podoplanin or 3851 of human podoplanin, plus C-terminus cysteine conjugated with keyhole limpet hemocyanin (KLH) with alum adjuvant (Imject Alum, Thermo Scientific Inc., Rockford, IL). The amino acids $38-51$ of human podoplanin are $100 \%$ homologous to the amino acids $38-51$ of monkey podoplanin. One week after the immunization, a secondary immunization was performed by an intraperitoneal booster injection 2 days before spleen cells were harvested. The spleen cells were fused with P3U1 cells using a Sendai virus (Hemagglutinating Virus of Japan: HVJ) envelope: GenomONE-CF (Ishihara Sangyo Kaisha, Ltd., Osaka, Japan) according to the manufacturer's instructions. The hybridomas were grown in RPMI medium with hypoxanthine, aminopterin and thymidine selection medium supplement (Invitrogen Corp., Carlsbad, CA). The culture supernatants were screened by enzyme-linked immunosorbent assay (ELISA) for binding to the synthetic peptide. The synthetic peptide GDGMVPPGIEDKIT (mpp3851) or EGGVAMPGAEDDVV (hpp3851), corresponding to amino acids $38-51$ of mouse podoplanin or $38-51$ of human podoplanin were immobilized on Maxisorp 96-well immunoplates (Thermo Fisher Scientific Inc.) at $1 \mu \mathrm{g} / \mathrm{ml}$ for $30 \mathrm{~min}$, respectively. After blocking with 1\% BSA in PBS, the plates were incubated with culture supernatant, followed by 1:1,000 diluted peroxidase-conjugated anti-rat IgG (Dako, Glostrup, Denmark). The enzymatic reaction was conducted with a substrate solution containing 3,3',5,5'tetramethylbenzidine (Thermo Fisher Scientific Inc.). The optical density was measured at $655 \mathrm{~nm}$ with a Benchmark microplate reader (Bio-Rad Laboratories Inc., Philadelphia, PA). These reactions were performed with a volume of 50 $\mu \mathrm{l}$ at $37^{\circ} \mathrm{C}$. The Hybridoma was further cultivated in CD Hybridoma medium (Life Technologies Japan Ltd.). The culture mediums were centrifuged, and the obtained supernatant was applied to a column of protein G-Sepharose (Thermo Scientific Inc., Rockford, IL).

\section{Immunohistochemistry}

This study used tissue of kidneys, lungs, tongues, and sublingual glands of eight-week-old male C57BL/6N mice $(n=5)(C L E A)$. The tissue was collected after euthanasia by intraperitoneal injection with sodium pentobarbital (10 $\mathrm{ml} / \mathrm{kg}$, Nembutal, Abbott Laboratories, North Chicago, IL). The protocol for the animal use was reviewed and approved by the animal experiment committee of Fukuoka Dental College in accordance with the principles of the Helsinki Declaration. Frozen $10 \mu \mathrm{m}$ sections of human sublingual glands from donated human bodies and mouse tissue were cut in a cryostat, and fixed in $100 \%$ methanol for $10 \mathrm{~min}$ at $-20^{\circ} \mathrm{C}$. The sections were treated with $0.1 \%$ goat serum for $30 \mathrm{~min}$ at $20^{\circ} \mathrm{C}$, and then the sections were treated with 0.5 $\mu \mathrm{g} / \mathrm{ml}$ of antibodies: monoclonal rat anti-mouse podoplanin (PMab-1), monoclonal hamster anti-mouse podoplanin (Mab-8.1.1, AngioBio Co., Del Mar, CA), monoclonal rat anti-human podoplanin (NZ-1.2), monoclonal mouse antihuman podoplanin (AB3, AngioBio), polyclonal rabbit anti-mouse $\alpha$-smooth muscle actin ( $\alpha$-SMA) (Epitomics, Burlingame, CA), and polyclonal rabbit anti-FLAG antibody, which recognizes DYKDDDDK (Sigma-Aldrich, St. Louis, MO) for $8 \mathrm{hr}$ at $4^{\circ} \mathrm{C}$. After the treatment with primary antibodies, the sections were immunostained for $0.5 \mathrm{hr}$ at $20^{\circ} \mathrm{C}$ with $0.1 \mu \mathrm{g} / \mathrm{ml}$ of second antibodies: Alexa Fluor (AF) 488 or 568-conjugated goat anti-mouse, goat anti-hamster, goat anti-rabbit, or goat anti-rat IgGs (Life Technologies Japan Ltd.). The nuclei were counterstained with DAPI (Sigma-Aldrich). The immunostained sections were mounted in $50 \%$ polyvinylpyrrolidone solution, and examined by fluorescence microscopy BZ-8100 (Keyence Corp., Osaka, Japan) or laser-scanning microscopy (LSM 710, Carl Zeiss, Jena, Germany) with a PlanApochromatic $60 \times$ oil immersion objective lens (numerical aperture $\times 1.3$ ). The CHO, CHO-mPDPN, HSC-3, HSC3-hPDPN, B16-F10, and LEC were cultured on cover slips in 6-well plates. The confluent monolayer of the cells on the cover slips was immersed in 4\% paraformaldehyde-PBS for $5 \mathrm{~min}$ at room temperature. After the treatment with $0.1 \%$ goat serum for $30 \mathrm{~min}$, the cells were immunostained as described above. The cover slips with the cells were mounted in $50 \%$ polyvinylpyrrolidone solution on slide glasses, and examined by laser-scanning microscopy. In the assessment of PMab-1 and NZ-1.2 about the specificities for mouse and human podoplanin, CHO-mPDPN and HSC3-hPDPN cells which are the transfectants of podoplanin gene, and B16-F10 and LEC cells expressing wild type podoplanin, were used as positive controls for reactivities to podoplanin on cultured cells while $\mathrm{CHO}$ and HSC3 were used as negative controls. The Mab-8.1.1 or AB3-positive kidney podocytes, lung epithelial cells, lymphatic vessels, and salivary gland myoepithelial cells were used as positive controls for reactivities to podoplanin on somatic tissue while the connective tissue, muscle cells, and blood vessels in tissue were used as negative controls.

To make paraffin-embedded sections, perfusion fixation was administered with $500 \mathrm{ml}$ of fixative solution containing 4\% formaldehyde-PBS for the eight-week-old male C57BL/6N mice after euthanasia by intraperitoneal injections with sodium pentobarbital. The dissected tissue was additionally fixed by immersion in a $10 \%$ formalinPBS solution, with a volume 50 times larger than the immersed tissue, for $8 \mathrm{hr}$ at room temperature as fixing of the tissue for longer than $24 \mathrm{hr}$ leads to masking or destruction of the podoplanin antigen. For the collection from the lungs, the fixative solution was injected into the euthanized mouse lungs by a syringe through the oral cavity to prevent the lung from collapsing by atmospheric pressure at the thoracotomy on the perfusion fixation. The $5-\mu \mathrm{m}$ thick paraffin-embedded sections were cut using a rotary microtome, mounted onto 3-aminopropyltriethoxysilane- 
coated slides, rehydrated in xylene and ethanol, and rinsed with deionized distilled water for $10 \mathrm{~min}$. Slides were immersed in $10 \mathrm{mM}$ sodium citrate buffer $(\mathrm{pH} \mathrm{6.0)}$ and boiled in a microwave oven for $10 \mathrm{~min}$ to break formalinformed protein cross-links masking the podoplanin antigen. After the boiling, the sections were rinsed by Phosphate buffered saline (PBS) and blocked with $0.1 \%$ goat serum. The immunostaining was performed using the first and second antibodies diluted into the blocking solution. All procedures were performed after washing with PBS for 30 min at room temperature. Also, the paraffin-embedded sections of monkey kidneys and lungs (HistoMap, Wako) were used to investigate the specificity of NZ-1.2 for the human podoplanin.

\section{Reverse transcription (RT)-PCR}

The total RNA extraction from $\mathrm{CHO}, \mathrm{CHO}-\mathrm{mPDPN}$, HSC-3, HSC3-hPDPN, B16-F10, and LEC was achieved with a QIAshredder column and an RNeasy kit (Qiagen, Inc., Tokyo, Japan). Contaminated genomic DNA was removed using DNAfree (Ambion, Huntingdon, UK), and the RT was performed on $30 \mathrm{ng}$ of total RNA, followed by 25-30 cycles of PCR for amplification using the Ex Taq hot start version (Takara Bio Inc., Otsu, Japan) with 50 pM of primer sets: mouse $\beta$-actin (forward, 5'-GTTCTAC AAATGTGGCTGAGGA; reverse, 5'-ATTGGTCTCAAG TCAGTGTACAG, $411 \mathrm{bp}$ ), mouse podoplanin coding sequence region (forward, 5'-CACCTCAGCAACCTCAGAC; reverse, 5'-AAGACGCCAACTATGATTCCAA, 192 bp), FLAG-tagged mouse podoplanin (forward, 5'-CACCTCA GCAACCTCAGAC; reverse, 5'-TGTCGTCATCGTCTTT GTAGTC, $284 \mathrm{bp}$ ), human $\beta$-actin (forward, 5'-ATGTTT GAGACCTTCAACAC; reverse, 5'-CACGTCACACTTC ATGATGG, 495 bp), human podoplanin (forward, 5'-TGA CAACTCTGGTGGCAACAAG; reverse, 5'-GGTCACTG TTGACAAACCATCTTT, 200 bp), and FLAG-tagged human podoplanin (forward, 5'-TGACAACTCTGGTGG CAACAAG; reverse, 5'-CGTCTTTGTAGTCGGGCGAG, $309 \mathrm{bp}$ ) where the specificities had been confirmed by the manufacturer (Sigma-Genosys Ltd., Cambridge, UK). The correct size of the amplified RT-PCR products were confirmed by electrophoresis on $2 \%$ agarose gel (NuSieve; FMC, Rockland, ME, USA) and visualized by Syber Green (Takara).

\section{Western-blot analysis}

The CHO, CHO-mPDPN, HSC-3, HSC3-hPDPN, B16-F10, and LEC cells forming a confluent monolayer in the 6-well plate were harvested by a cell scraper, and solubilized in $100 \mu \mathrm{l}$ of cell lysis buffer (Cell Signaling Technology, Inc., Danvers, MA). The lysate of whole cell protein $(1 \mathrm{mg} / \mathrm{ml})$ was mixed with an equal volume of sample buffer (Bio-Rad Laboratories Inc., Hercules, CA). A $20-\mu$ l volume of sample was loaded on $15 \%$ polyacrylamide gel by electrophoresis with Mini-protean III (Bio$\mathrm{Rad})$, and the separated proteins were transferred onto a
A
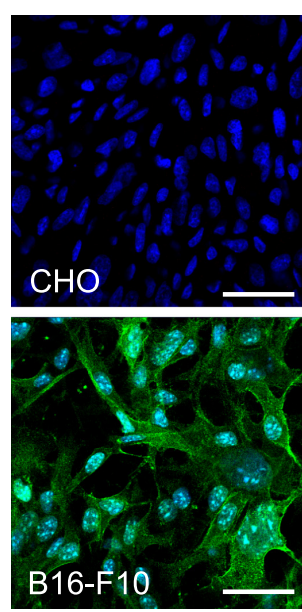

PMab-1-FLAG
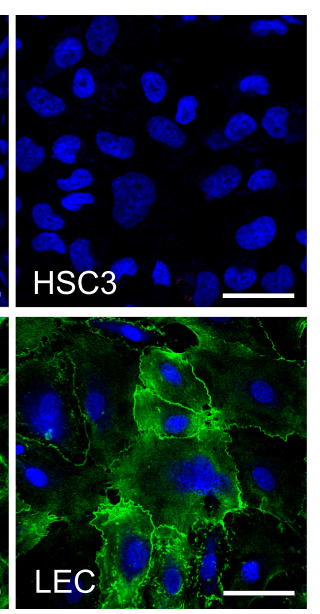

NZ-1.2-FLAG
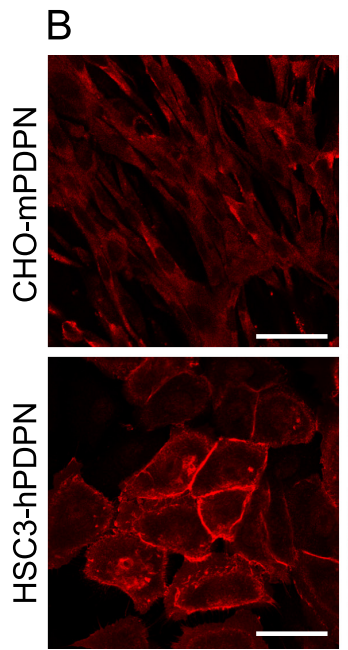

FLAG
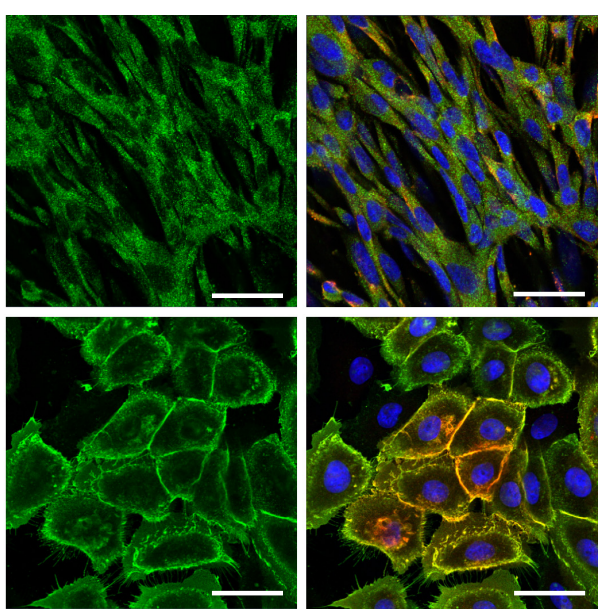

PMab-1/NZ-1.2

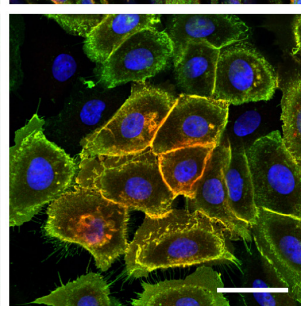

merge

Fig. 1. (A) Immunostaining for podoplanin and FLAG on cultured cells. The CHO and B16-F10 cells were exposed to a cocktail of PMab-1 (green) and anti-FLAG (red) (PMab-1-FLAG). The HSC3 and LEC cells were exposed to a cocktail of NZ-1.2 (green) and anti-FLAG (NZ-1.2FLAG). The nuclei were counterstained with DAPI. The CHO was not stained by PMab-1 or anti-FLAG. The B16-F10 was stained by PMab-1 at the cytoplasm but not by anti-FLAG. The HSC3 was not stained by NZ-1.2 or anti-FLAG but LEC was stained by NZ-1.2 at the cell-cell junction and the cytoplasm. (B) Immunostaining of the transfectants for mouse and human podoplanin-FLAG genes. The CHO-mPDPN cells were exposed to a cocktail of PMab-1 (green) and anti-FLAG (red). The HSC3-hPDPN cells were exposed to a cocktail of NZ-1.2 (green) and anti-FLAG. The nuclei were counterstained using DAPI. The FLAG protein expressed on CHO-mPDPN and HSC3-hPDPN were stained by anti-FLAG. The mouse or human podoplanin expressed on CHO-mPDPN or HSC3-hPDPN were stained by PMab-1 or NZ-1.2. The merged images indicate that the PMab-1 or NZ-1.2-reacting region on CHO-mPDPN or HSC3-hPDPN coincided with the anti-FLAG reacting region. Nuclei were counterstained using DAPI. Bar $=50 \mu \mathrm{m}$. 
PVDF membrane (Life Technologies Japan Ltd.). After blocking of the PVDF membrane by 5\% skim milk for $3 \mathrm{hr}$ at $20^{\circ} \mathrm{C}$, the membrane was treated with the blocking agent containing PMab-1 and NZ-1.2 $(10 \mu \mathrm{g} / \mathrm{ml})$ for $2 \mathrm{hr}$ at $20^{\circ} \mathrm{C}$, and with peroxidase-conjugated goat anti-rat $(1 \mu \mathrm{g} / \mathrm{ml})$ for $1 \mathrm{hr}$ at $20^{\circ} \mathrm{C}$, and then visualized by the substrate of a Vector Elite $\mathrm{ABC}$ kit and a Vector VIP kit (Vector Laboratories, Burlingame, CA) or Immun-Star WesternC Chemiluminescence kit (Bio-Rad), and scanned by an image analyzer LAS-3000 mini (Fujifilm Corp., Tokyo, Japan).

\section{Results}

Examination of anti-mouse podoplanin monoclonal antibody PMab-1 and anti-human podoplanin monoclonal antibody NZ-1.2 against cultured cells

The hybridoma clones producing anti-mouse podoplanin monoclonal antibody PMab-1 ( $\operatorname{IgG}_{2 \mathrm{a}}$ subclass) or antihuman monoclonal antibody NZ-1.2 ( $\operatorname{IgG}_{2 \mathrm{a}}$ subclass) were established. In the immunocytochemical analysis on cul-

\section{A}

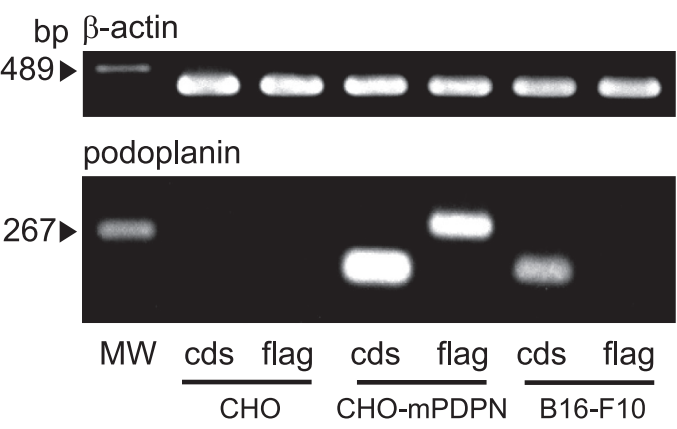

B

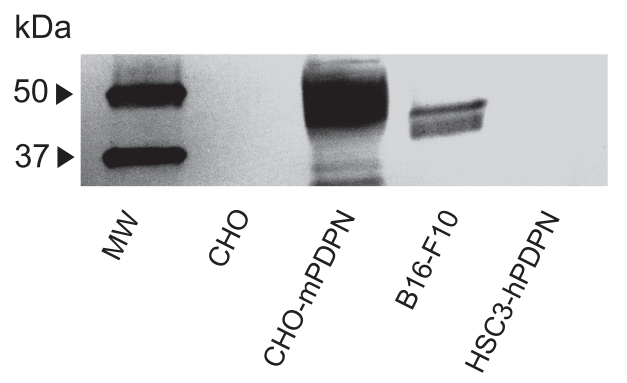

tured cells, $\mathrm{CHO}$ was not immunostained by PMab-1 or anti-FLAG, whereas B16-F10 was stained by PMab-1 (Fig. 1). The HSC3 was not immunostained by NZ-1.2 or anti-FLAG, while LEC was stained by NZ-1.2 at the cellcell junction and the cytoplasm. The CHO-mPDPN, which is the stable transfectant of CHO-K1 with FLAG-tagged mouse podoplanin gene, was stained by PMab-1 and antiFLAG (Fig. 1). The HSC3-hPDPN, which is the stable transfectant of HSC3 with the FLAG-tagged human podoplanin gene, was stained by NZ-1.2 and anti-FLAG. The stained region by PMab-1 or NZ-1.2 on CHO-mPDPN or HSC3-hPDPN coincided with the anti-FLAG reaction.

The PCR products for the mouse podoplanin gene coding sequence (CDS) and the FLAG-tagged mouse podoplanin gene were not detected in $\mathrm{CHO}$, but detected in CHO-mPDPN (Fig. 2). The PCR products of podoplanin CDS were detected solely in the mouse melanoma B16-F10, which expresses intrinsic podoplanin. Western-blot analysis showed that PMab-1 did not react to the whole cell protein from $\mathrm{CHO}$ or HSC3-hPDPN, whereas about 40-kDa protein was detected in CHO-mPDPN and B16-F10 (Fig. 2). The

C

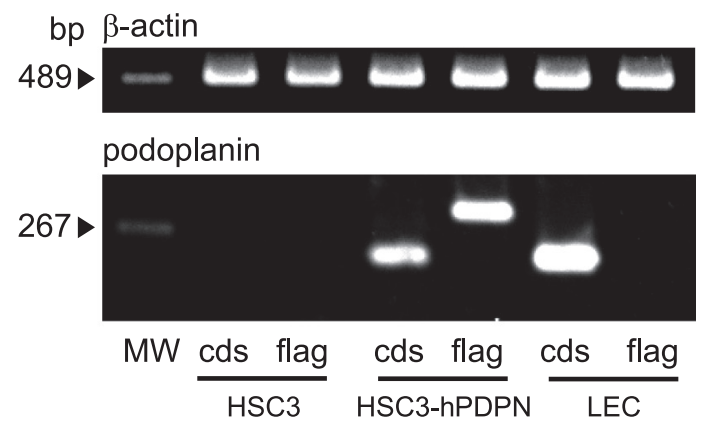

D

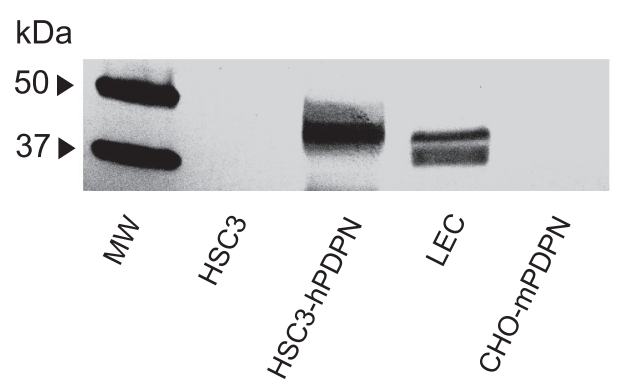

Fig. 2. Gene and protein analysis for podoplanin on cultured cells. (A) RT-PCR analysis on the mouse podoplanin mRNA expression. The PCR products of mouse podoplanin gene coding sequence (cds) and the FLAG-tagged mouse podoplanin gene (flag) were not detected from the CHO, but were detected from CHO-mPDPN. The PCR products of podoplanin CDS were also detected from B16-F10. MW, molecular weight marker. (B) Western-blot analysis of the mouse podoplanin protein. The PMab-1 did not react to the whole cell protein from CHO or HSC3-hPDPN, whereas about an 40-kDa protein was detected from CHO-mPDPN and B16-F10. The molecular weight of the FLAG-tagged podoplanin in CHO-mPDPN is slightly larger than the intrinsic podoplanin of about $40 \mathrm{kDa}$ in B16-F 10 because of the molecular weight of the FLAG. MW, molecular weight marker. (C) RT-PCR analysis for the human podoplanin mRNA expression. The PCR products of the human podoplanin coding sequence (cds) and the FLAG-tagged human podoplanin gene (flag) were not detected from HSC3, but were detected from HSC3-hPDPN. The PCR products of podoplanin CDS were also detected from LEC. MW, molecular weight marker. (D) Western-blot analysis of the human podoplanin protein. The NZ-1.2 did not react to the whole cell protein from HSC3 or CHO-mPDPN, whereas an about 40-kDa protein was detected from the HSC3-hPDPN and LEC. The molecular weight of the FLAG-tagged podoplanin from HSC3-hPDPN is slightly larger than the intrinsic podoplanin of about $40 \mathrm{kDa}$ from LEC. MW, molecular weight marker. 
molecular weight of FLAG-tagged podoplanin in CHOmPDPN is slightly larger than the intrinsic podoplanin in B16-F10. The PCR products of human podoplanin CDS and the FLAG-tagged human podoplanin gene were not detected in HSC3, but detected in HSC3-hPDPN (Fig. 2). The PCR products of human podoplanin CDS were detected in LEC, which expresses intrinsic podoplanin. Western-blot analysis showed that NZ-1.2 did not react with the whole cell protein from HSC3 or CHO-mPDPN, whereas about a 40-kDa protein was detected in HSC3-hPDPN and LEC (Fig. 2). The molecular weight of the FLAG-tagged human podoplanin from HSC3-hPDPN is slightly larger than the intrinsic podoplanin from LEC.

\section{Immunohistochemical analysis of PMab-1 and NZ-1.2 on the mouse and human tissues}

In the immunostaining for podoplanin of the mouse kidney, PMab-1-reacting region on the glomerular capillary coincided with another anti-mouse podoplanin Mab-8.1.1reacting region in the glomerulus of the renal cortex without cross-reaction to the uriniferous tubules (Fig. 3). In the immunostaining for podoplanin of the mouse lung, the PMab-1-reacting region on pulmonary alveoli and the pulmonary pleura coincided with the Mab-8.1.1-reacting region, with a reaction stronger than PMab-1. Immunostaining for $\alpha$-SMA and podoplanin of mouse tongue, the anti- $\alpha$-SMA reacted to blood vessels, whereas the PMab-1 reacted to lymphatic vessels in the tunica muscularis (Fig. 4). In the mouse lingual gland, reaction products with PMab-1 were observed on acini and on the duct, and on lymphatic vessels without cross-reaction to any tissue, while anti- $\alpha$-SMA reacted to the secretory terminal end pieces of acini and on the duct with cross-reaction to intraacinar components and to lingual mucous epithelia (Fig. 4). In the mouse sublingual gland, the terminal secretory end pieces were densely composed of large mucous-filled cells (Fig. 5). The intercalated and striated ducts were composed of columnar cells with intensely acidopillic cytoplasm and well developed when compared with the lingual gland. In the immunostaining against podoplanin and $\alpha$-SMA of the mouse sublingual glands, the regions of myoepithelial cells around acini and the basal side of intercalated and striated ducts reacted with anti- $\alpha$-SMA, and Mab-8.1.1 or PMab-1. The cell-cell junction of acinar cells and the luminal side of intercalated ducts, where myoepithelial cells are absent, were immunostained by anti- $\alpha$-SMA, but not by Mab-8.1.1 or PMab-1. The Mab-8.1.1 reacted to almost the same region as that stained by PMab-1, but also showed a weak cross-reaction with connective tissue around the striated duct. In the human sublingual gland, the terminal secretory end pieces of acini were densely composed of large mucous-filled cells with a few serous pyramidal cells (Fig. 6). In the immunostaining for podoplanin and $\alpha$-SMA of human sublingual glands, the regions of myoepithelial cells around mucous acini reacted by anti- $\alpha$-SMA, antihuman podoplanin AB3, and NZ-1.2 while the basal portion of serous acini partially showed a weak reaction to the antibodies. The cell-cell junction of acinar cells and the luminal side of ducts, where myoepithelial cells were absent, were immunostained by anti- $\alpha-\mathrm{SMA}$, but not by

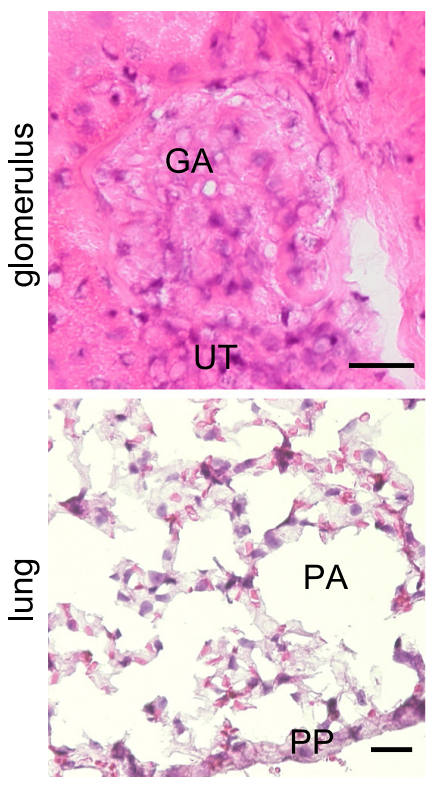

$\mathrm{HE}$
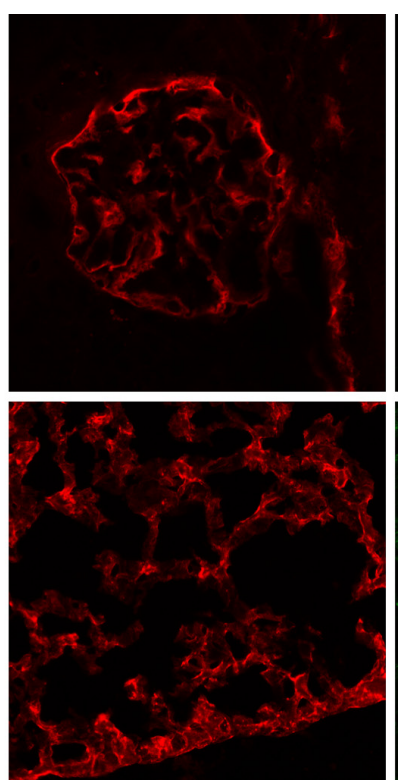

8.1.1
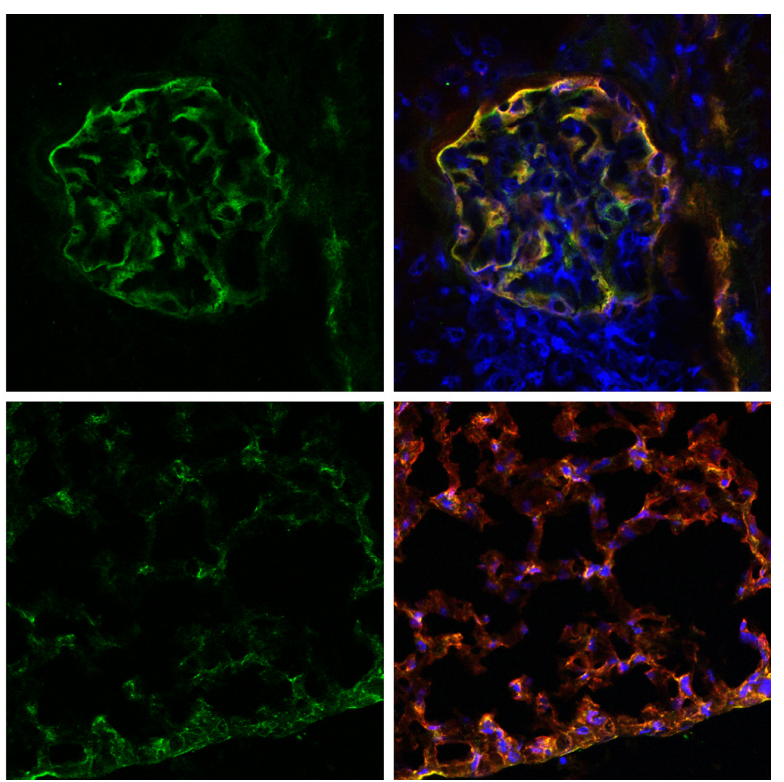

PMab-1

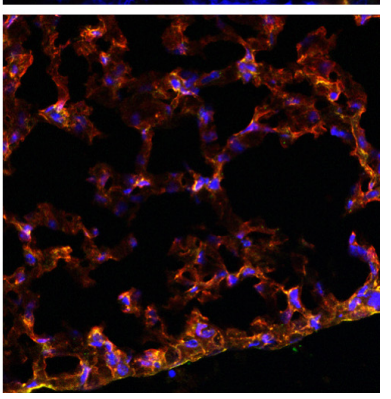

merge

Fig. 3. Immunostaining for podoplanin on mouse kidney and lung tissue. In the glomerulus of the renal cortex, the merged image indicates that the PMab-1-reacting region on the glomerular capillary (GA) (green) coincides with the Mab-8.1.1-reacting region (red). The PMab-1 and Mab-8.1.1 did not react to uriniferous tubules (UT). In the lung, the merged image indicates that the pulmonary pleura (PP) and a part of the pulmonary alveoli (PA) were immunostained by PMab-1 (green). The immunoreaction of Mab-8.1.1 (red) to alveolar cells was stronger than PMab-1 and the Mab-8.1.1 specificity was broader comparing with PMab-1. Nuclei were counterstained using DAPI. Bar=20 $\mu$ m. 

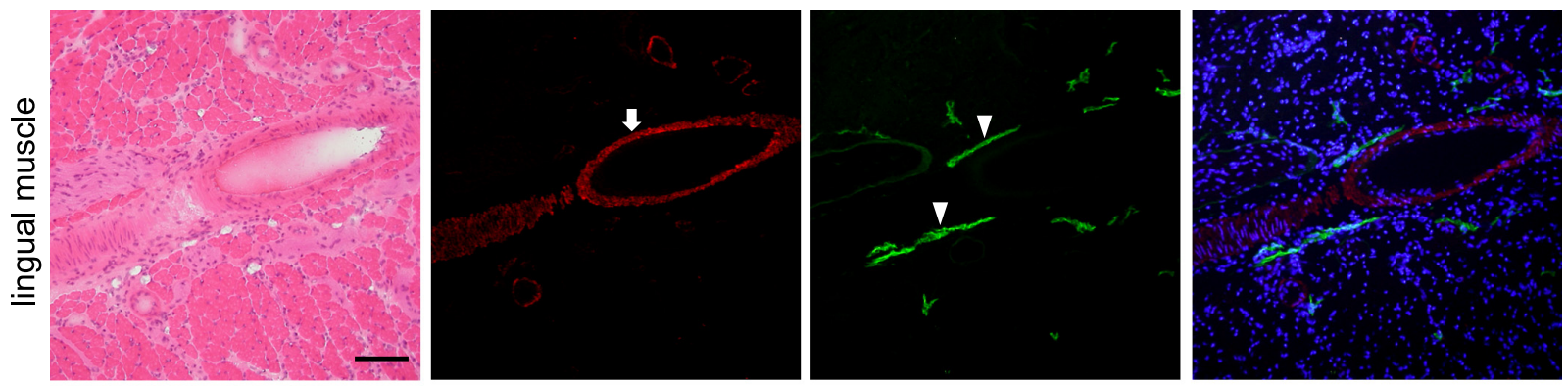

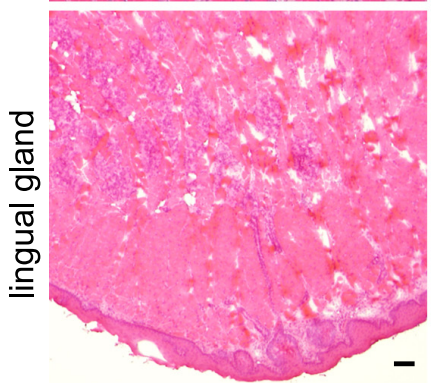

HE

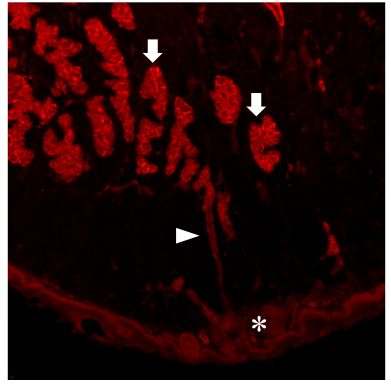

SMA

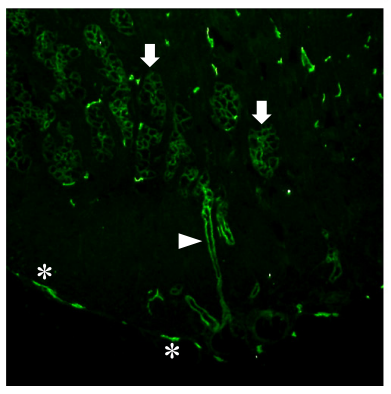

PMab-1

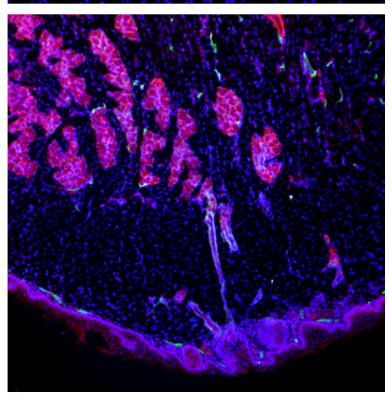

merge

Fig. 4. Immunostaining for podoplanin on mouse radix linguae tissue. In the tunica muscularis, blood vessels were stained by anti- $\alpha$-SMA (arrow) (red), whereas lymphatic vessels were stained by PMab-1 (arrowheads) (green). In the lingual gland reaction products with PMab-1 (green) were also observed on acini (arrows) and on the duct (arrowhead), and on lymphatic vessels (asterisks) without cross reaction to any tissue, while reaction products with anti- $\alpha$-SMA (red) were observed on the secretory terminal end pieces of acini (arrows) and on the duct (arrowhead) with cross reaction to the intra-acinar components and to lingual mucous epithelia (asterisk). Nuclei were counterstained using DAPI. Bar $=100 \mu \mathrm{m}$.
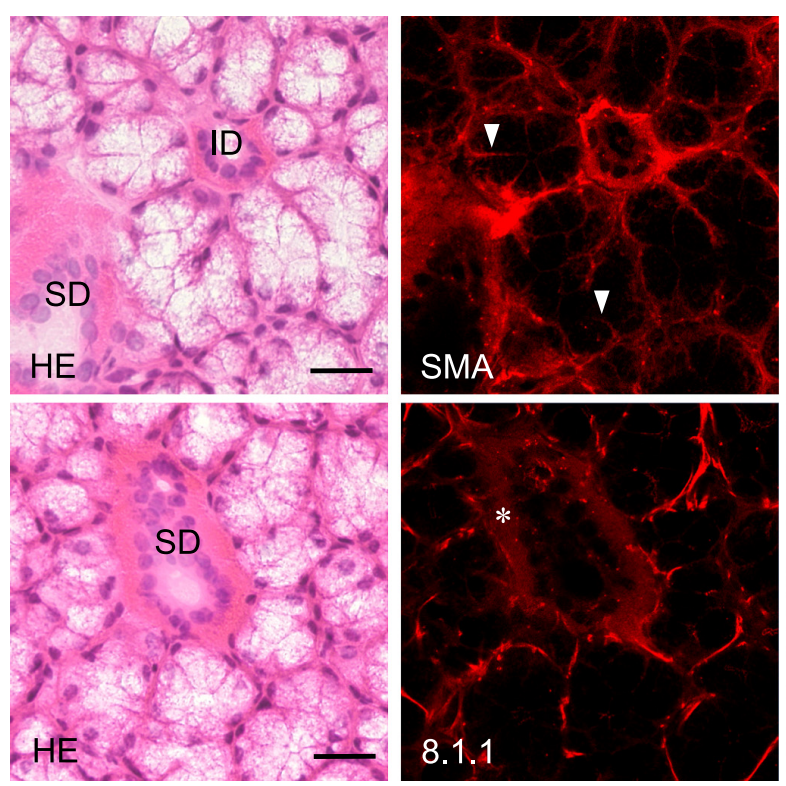
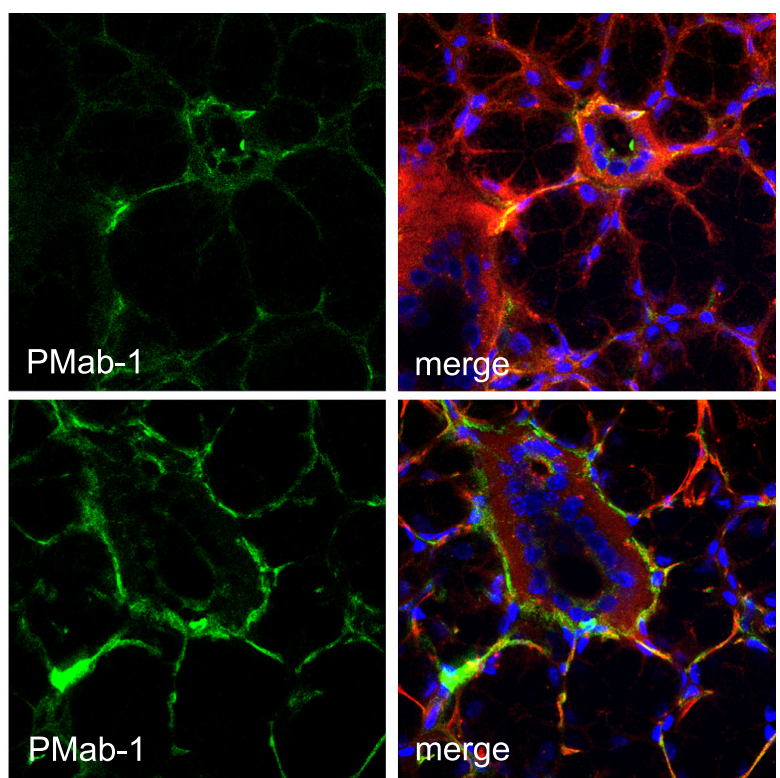

Fig. 5. Immunostaining for podoplanin on mouse sublingual gland tissue. The H-E staining shows that the secretory terminal end pieces of acini are composed of mucous-filled acinar cells. The intercalated duct (ID) is found interposed between acinar cells, and has a narrow lumen surrounded by cuboidal cells. The striated duct (SD) is composed of columnar cells with acidopillic cytoplasm. The regions of the myoepithelial cells around acini and the basal side of the intercalated and striated ducts reacted by anti- $\alpha$-SMA (red), anti-podoplanin Mab-8.1.1 (red), and PMab-1 (green). The cell-cell junction of the acinar cells where myoepithelial cells are not present were immunostained with anti- $\alpha$-SMA (arrowheads) but not with Mab-8.1.1 or PMab-1. The Mab-8.1.1 reacted more strongly to the PMab-1-reacting region, but also showed a weak cross reaction with connective tissue around the striated duct (asterisk). Nuclei were counterstained by DAPI. Bar $=100 \mu \mathrm{m}$. 

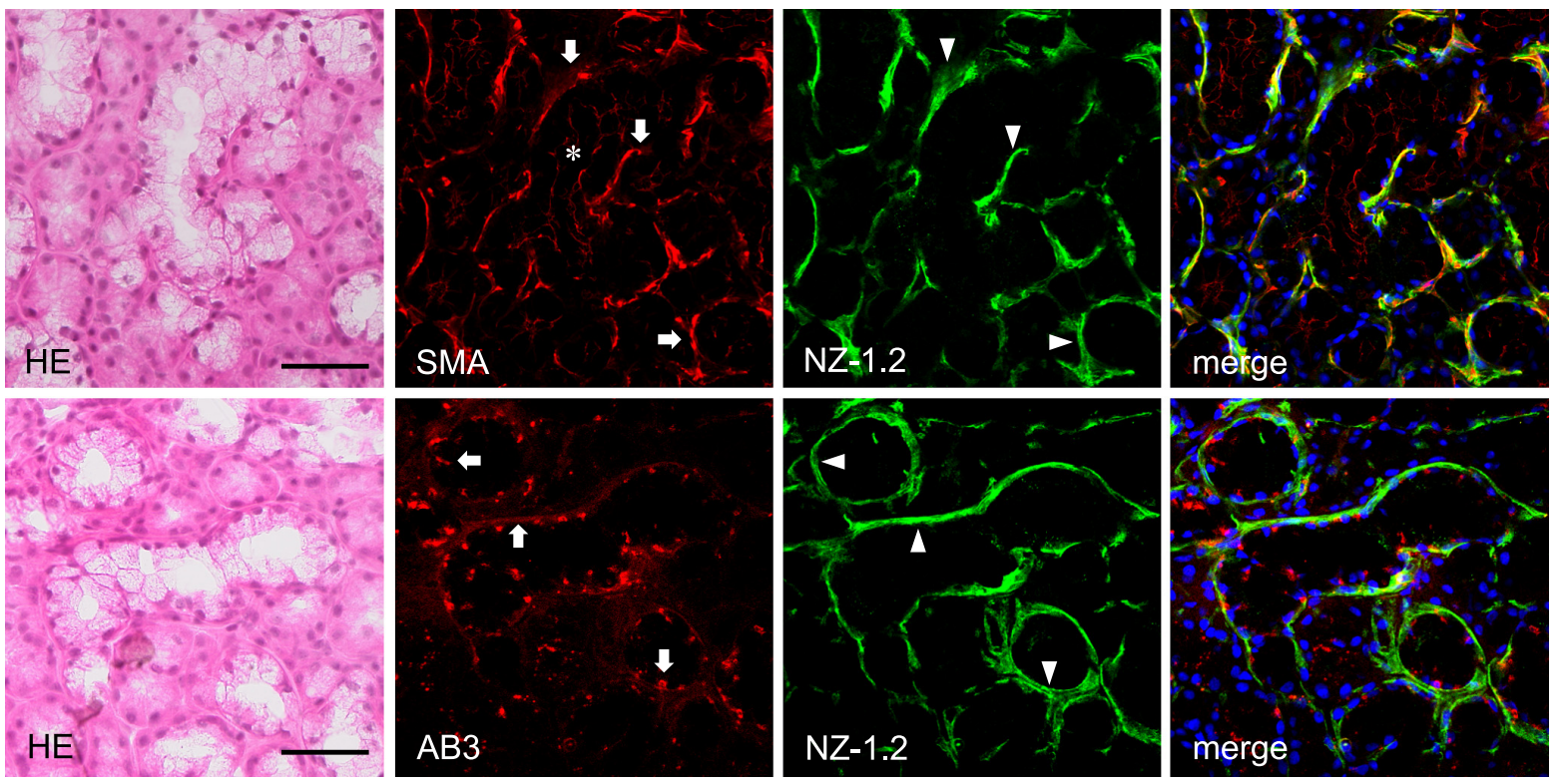

Fig. 6. Immunostaining for podoplanin on human sublingual gland tissue. The H-E staining shows the secretory terminal end pieces which composed of large mucous-filled acinar cells, and a few serous pyramidal cells stained to a stronger extent than the mucous acini. The regions of myoepithelial cells around mucous acini reacted by anti- $\alpha$-SMA (arrows) (red), AB3 (arrows) (red), and NZ-1.2 (arrowheads) (green) while the basal portion of serous acini adjacent to mucous acini partially showed a weak reaction to the antibodies. The cell-cell junctions of acinar cells where myoepithelial cells are absent were also stained by anti- $\alpha$-SMA (asterisk), but not with AB3 or NZ-1.2. The AB3 reacted to the NZ-1.2-staining region to a strikingly weaker extent and also showed a cross-reaction with nuclei. Nuclei were counterstained using DAPI. Bar $=100 \mu \mathrm{m}$.
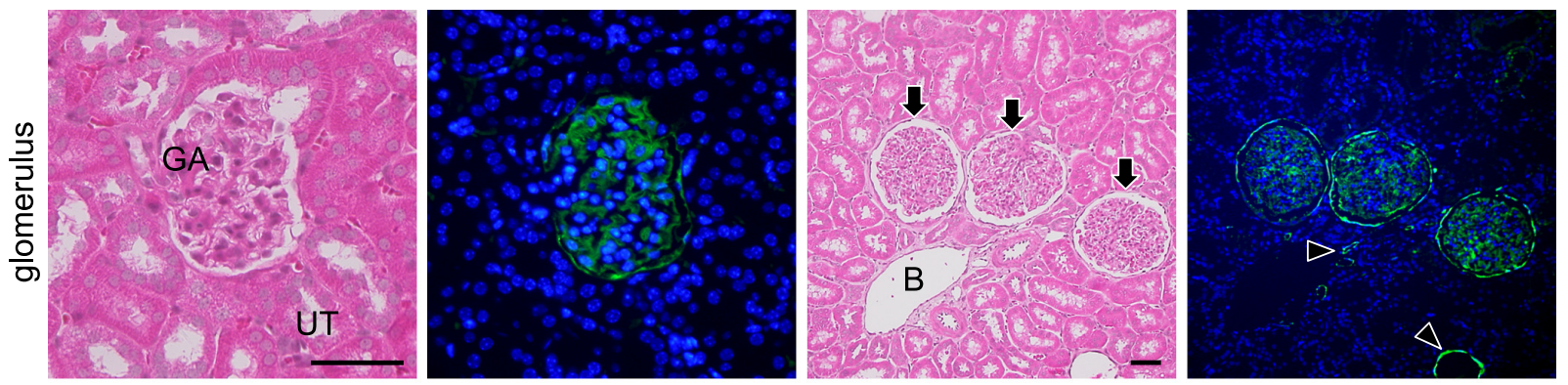

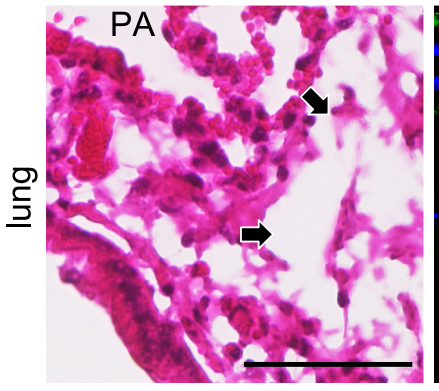

HE

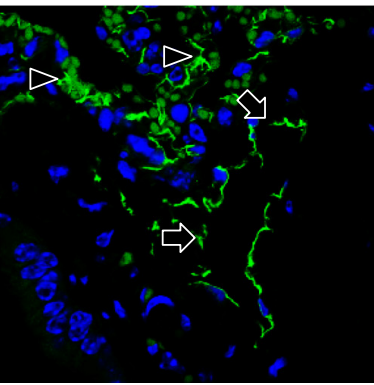

PMab-1

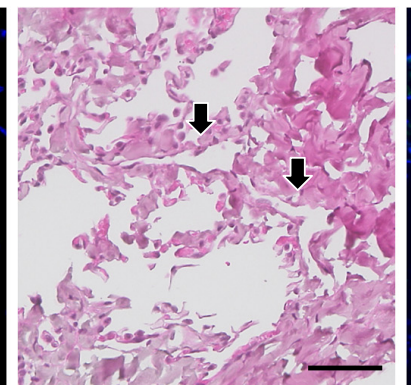

HE

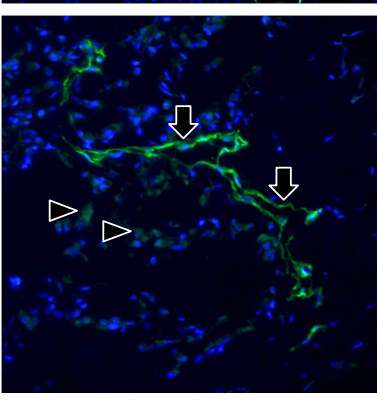

NZ-1.2

mouse

monkey

Fig. 7. The immunostaining of the paraffin-embedded sections by PMab-1 and NZ-1.2. The PMab-1 reacted on the glomerulus (GA) of the renal cortex without any cross reaction to uriniferous tubules (UT) in the mouse kidney, and reacted on the pulmonary alveoli (PA, arrowheads) and lymphatic vessels (arrows) in the mouse lungs. The NZ-1.2 reacted on the glomerulus (arrows) of the renal cortex and lymphatic vessels (arrowheads), but not on the blood vessels (B) in the monkey kidney, and reacted on the pulmonary alveoli (arrowheads) and lymphatic vessels (arrows) in the monkey lung. Bar=50 $\mu \mathrm{m}$. 
$\mathrm{AB} 3$ or NZ-1.2. The AB3 reacted to the NZ-1.2-staining region to a strikingly weaker extent; and also showed a cross-reaction with nuclei. In the immunostaining of paraffin-embedded sections, PMab-1 reacted to the mouse kidney glomerulus, and to the mouse pulmonary alveoli and lung lymphatic vessels (Fig. 7). The NZ-1.2 also reacted to the monkey glomerulus and kidney lymphatic vessels, and to the monkey pulmonary alveoli and lung lymphatic vessels.

\section{Discussion}

A rat monoclonal antibody NZ-1, which is specific to human podoplanin has previously been established [11], and NZ-1 has enabled neutralized human podoplanininduced platelet aggregation and pulmonary metastasis [12]. The NZ-1 antibody is also useful in immunohistochemistry, suggesting that the NZ-1 immunogen is very effective in producing anti-podoplanin antibodies. However, NZ-1 does not react with mouse, rat, or hamster podoplanin (data not shown). For analyzing mouse podoplanin expression in vivo, the investigation reported here intended to produce a novel anti-mouse podoplanin by immunizing rats with amino acids $38-51$ of mouse podoplanin, which corresponds to the NZ-1 immunogen. It was possible to establish both an anti-mouse podoplanin antibody (PMab-1) and an anti-human podoplanin antibody (NZ-1.2), which are very useful in immunohistochemistry. In the analysis using cultured cells, $\mathrm{CHO}$ was not immunostained by PMab-1 or anti-FLAG; B16-F10 was stained by PMab-1; and CHOmPDPN was stained by PMab-1 and anti-FLAG, suggesting that the reaction of PMab-1 is specific for the mouse podoplanin-expressing cells (Fig. 1). We have previously reported the gene and protein expression of podoplanin in the cultured human lymphatic endothelial cell LEC [2426]. The study here showed that LEC was stained by NZ1.2. Further, HSC3 was not immunostained by NZ-1.2 or anti-FLAG, whereas the PMab-1 or NZ-1.2-reacting region on CHO-mPDPN or HSC3-hPDPN coincided with the anti-FLAG-reacting region, suggesting that the reaction of PMab-1 or NZ-1.2 is specific for mouse or human podoplanin (Fig. 1). The specificity of PMab-1 or NZ-1.2 was also confirmed by Western-blot analysis (Fig. 2). The mouse podoplanin gene and protein were not detected from $\mathrm{CHO}$, but were detected from CHO-mPDPN and from mouse melanoma B16-F10 expressing intrinsic podoplanin. The human podoplanin gene and protein were not detected from HSC3, but was detected from HSC3-hPDPN and from human lymphatic endothelial cell LEC expressing intrinsic podoplanin.

Podoplanin is known as an antigen of podocytes of kidney [1]. It is thought that podoplanin plays a role in maintaining the shape of podocyte foot processes and glomerular permeability, because podocyte foot processes are extensive flattenings, and podoplanin is selectively reduced to $<30 \%$ in a rat model of nephropathy by puromycin aminonucleoside nephrosis accompanying severe pro- teinuria [15]. Podoplanin is also well known as a lymphatic endothelial cell marker, and is homologous to the T1 $\alpha-2$ gene, which encodes the type I alveolar cell specific antigen $[2,23,35]$. Podoplanin ${ }^{-1}$ mice have defects in the lymphatic formation with congenital lymphedema, but not have defects in the blood vessel pattern formation, and they die at birth because of the respiratory failure [27]. In the immunohistochemical study on podoplanin expression of mouse kidneys here, the PMab-1-reacting region on the glomerular capillary coincided with the Mab-8.1.1-reacting region in the glomerulus of the renal cortex without crossreaction to the uriniferous tubules (Fig. 3). The reaction intensity for the glomerulus of PMab-1 is similar to that of Mab-8.1.1, suggesting that PMab-1 is a useful antibody to mouse podoplanin as well as Mab-8.1.1. In the immunostaining for podoplanin of mouse lungs, the pulmonary pleura and a part of the pulmonary alveoli were immunostained by PMab-1 and Mab-8.1.1 (Fig. 3). The immunoreaction of Mab-8.1.1 to alveolar cells was stronger and broader than that of PMab-1. In the immunostaining for $\alpha$-SMA and podoplanin of mouse tongues, anti- $\alpha$-SMA reacted to blood vessels, whereas PMab-1 reacted to lymphatic vessels in the tunica muscularis, indicating that PMab-1 is also useful to discriminate lymphatic vessels from blood vessels in mouse somatic tissue (Fig. 4).

Immunohistochemical studies have recently demonstrated that podoplanin is a salivary gland myoepithelial cell antigen [4, 5, 20]. In this study of mouse lingual glands, PMab-1 reacted to acini, duct, and lymphatic vessels without cross-reaction to other tissue, while anti- $\alpha$-SMA reacted to acini and ducts with cross-reaction to intra-acinar components and lingual mucous epithelia (Fig. 4). Further, in the mouse sublingual glands, the regions of myoepithelial cells around acini (Fig. 5) were immunostained by anti- $\alpha$ SMA, Mab-8.1.1, and PMab-1. The Mab-8.1.1 reacted more strongly to the PMab-1-reacting region but also showed a weak cross-reaction with connective tissue around the striated duct, suggesting that PMab-1 could be a useful antibody to identify podoplanin on salivary gland myoepithelial cells. The cell-cell junction of acinar cells and the luminal side of the intercalated duct, where myoepithelial cells are absent, were immunostained by anti- $\alpha$-SMA but not by Mab-8.1.1 or PMab-1, suggesting that Mab-8.1.1 and PMab-1 are superior to anti- $\alpha$-SMA in identifying myoepithelial cells.

In human sublingual glands, $\alpha$-SMA-positive myoepithelial cells around mucous acini were immunostained by the anti-human podoplanin antibodies NZ-1.2 and AB3, while the basal portion of serous acini partially showed a weak reaction to the antibodies (Fig. 6). Myoepithelial cell shape varies considerably with the different glands in relation to the physical properties of secretions. In the parotid gland few myoepithelial cells are on the terminal portion of serous acini while in the sublingual gland, approximately $50 \%$ of the terminal portion surface was covered by myoepithelial cells with a number of broad and extensive cellular processes on the mucous acini, and with a few 
thin processes on the serous acini [19]. These coincide with the immunostaining of acini of sublingual gland by NZ-1.2, suggesting the specificity of NZ-1.2 for human podoplanin on the myoepithelial cells. The cell-cell junctions of acinar cells where myoepithelial cells are absent were also immunostained by anti- $\alpha$-SMA but not by AB3 or NZ-1.2. The NZ-1.2 reacted to a very similar region as that stained by $A B 3$ and to a much higher extent, and ABS showed a cross-reaction with nuclei, suggesting that NZ-1.2 is more sensitive and highly specific against the podoplanin of human myoepithelial cells when compared with AB3.

The immunostaining of paraffin-embedded sections showed a reaction with PMab-1 to the mouse kidney glomerulus, pulmonary alveoli, and lung lymphatic vessels without any cross reactions (Fig. 7). Further, the immunostaining of paraffin-embedded sections showed the reaction of NZ-1.2 to monkey glomerulus and kidney lymphatic vessels, and to monkey pulmonary alveoli and lung lymphatic vessels. The epitope of NZ-1.2 is the amino acids 38-51 of the human podoplanin (Accession No. AB127958) which is $100 \%$ homologous to amino acids $38-51$ of the monkey podoplanin (Accession No. XM_001106933.2.). These suggest that PMab-1 and NZ-1.2 are useful for the immunostaining for podoplanin on paraffin-embedded sections of mouse and human/monkey as well as on frozen sections.

In conclusion, rat monoclonal antibodies PMab- 1 to mouse podoplanin and NZ-1.2 to human podoplanin were established. These antibodies were shown to be useful for both immunostaining and Western-blot.

\section{Acknowledgments}

We thank Ryo Yanagiya, Shunpei Morita, Imi Saito, and Junko Aita for their excellent technical assistance. This work was supported in part by Grant-in-Aid for Young Scientists (B) 23701043 and 23791584 (Kaneko and Kato), Scientific Research (B) 22390345 (principal: Sawa), and Exploratory Research 23659884 (principal: Sawa) from Japan Society for the Promotion of Science, by Mitsubishi Pharma Research Foundation (Kato and Kaneko), by Children Cancer Association of Japan (Kato), by Office for Gender Equality of Yamagata University (Kaneko), and by Intelligent Cosmos Academic Foundation (Kato).

\section{References}

1. Breiteneder-Geleff, S., Matsui, K., Soleiman, A., Meraner, P., Poczewski, H., Kalt, R., Schaffner, G. and Kerjaschki, D. (1997) Podoplanin, novel43-kd membrane protein of glomerular epithelial cells, is down-regulated in puromycin nephrosis. Am. J. Pathol. 151; 1141-1152.

2. Dobbs, L. G., Williams, M. C. and Gonzalez, R. (1988) Monoclonal antibodies specific to apical surfaces of rat alveolar type I cells bind to surfaces of cultured, but not freshly isolated, type II cells. Biochim. Biophys. Acta 970; 146-156.

3. Hantusch, B., Kalt, R., Krieger, S., Puri, C. and Kerjaschki, D. (2007) Sp1/Sp3 and DNA-methylation contribute to basal transcriptional activation of human podoplanin in MG63 versus Saos-2 osteoblastic cells. BMC Mol. Biol. 8; 20.

4. Hata, M., Ueki, T., Sato, A., Kojima, H. and Sawa, Y. (2008) Expression of podoplanin in the mouse salivary glands. Arch. Oral Biol. 53; 835-841.

5. Hata, M., Amano, I., Tsuruga, E., Kojima, H. and Sawa, Y. (2010) Immunoelectron microscopic study of podoplanin localization in mouse salivary gland myoepithelium. Acta Histochem. Cytochem. 43; 77-82.

6. Imaizumi, Y., Amano, I., Tsuruga, E., Kojima, H. and Sawa, Y. (2010) Immunohistochemical examination for the distribution of podoplanin-expressing cells in developing mouse molar tooth germs. Acta Histochem. Cytochem. 43; 115-121.

7. Kaneko, M., Kato, Y., Kunita, A., Fujita, N., Tsuruo, T. and Osawa, M. (2004) Functional sialylated O-glycan to platelet aggregation on Aggrus (T1alpha/Podoplanin) molecules expressed in Chinese hamster ovary cells. J. Biol. Chem. 279; 38838-38843.

8. Kaneko, M. K., Kato, Y., Kitano, T. and Osawa, M. (2006) Conservation of a platelet activating domain of Aggrus/podoplanin as a platelet aggregation-inducing factor. Gene 378; 52-57.

9. Kaneko, M. K., Kato, Y., Kameyama, A., Ito, H., Kuno, A., Hirabayashi, J., Kubota, T., Amano, K., Chiba, Y., Hasegawa, Y., Sasagawa, I., Mishima, K. and Narimatsu, H. (2007) Functional glycosylation of human podoplanin: glycan structure of platelet aggregation-inducing factor. FEBS Lett. 581; 331-336.

10. Kato, Y., Fujita, N., Kunita, A., Sato, S., Kaneko, M. and Osawa, M. (2003) Molecular identification of Aggrus/T1 $\alpha$ as a platelet aggregation-inducing factor expressed in colorectal tumors. $J$. Biol. Chem. 278; 51599-51605.

11. Kato, Y., Kaneko, M. K., Kuno, A., Uchiyama, N., Amano, K., Chiba, Y., Hasegawa, Y., Hirabayashi, J., Narimatsu, H., Mishima, K. and Osawa, M. (2006) Inhibition of tumor cellinduced platelet aggregation using a novel anti-podoplanin antibody reacting with its platelet-aggregation-stimulating domain. Biochem. Biophys. Res. Commun. 349; 1301-1307.

12. Kato, Y., Kaneko, M. K., Kunita, A., Ito, H., Kameyama, A., Ogasawara, S., Matsuura, N., Hasegawa, Y., Suzuki-Inoue, K., Inoue, O., Ozaki, Y. and Narimatsu, H. (2008) Molecular analysis of the pathophysiological binding of the platelet aggregationinducing factor podoplanin to the C-type lectin-like receptor CLEC-2. Cancer Sci. 99; 54-61.

13. Kawaguchi, H., El-Naggar, A. K., Papadimitrakopoulou, V., Ren, H., Fan, Y. H., Feng, L., Lee, J. J., Kim, E., Hong, W. K., Lippman, S. M. and Mao, L. (2008) Podoplanin: a novel marker for oral cancer risk in patients with oral premalignancy. J. Clin. Oncol. 26; 354-360.

14. Kimura, N. and Kimura, I. (2005) Podoplanin as a marker for mesothelioma. Pathol. Int. 55; 83-86.

15. Koop, K., Eikmans, M., Wehland, M., Baelde, H., Ijpelaar, D., Kreutz, R., Kawachi, H., Kerjaschki, D., de Heer, E. and Bruijn, J. A. (2008) Selective loss of podoplanin protein expression accompanies proteinuria and precedes alterations in podocyte morphology in a spontaneous proteinuric rat model. Am. J. Pathol. 173; 315-326.

16. Kunita, A., Kashima, T. G., Morishita, Y., Fukayama, M., Kato, Y., Tsuruo, T. and Fujita, N. (2007) The platelet aggregationinducing factor aggrus/podoplanin promotes pulmonary metastasis. Am. J. Pathol. 170; 1337-1347.

17. Marsee, D. K., Pinkus, G. S. and Hornick, J. L. (2009) Podoplanin (D2-40) is a highly effective marker of follicular dendritic cells. Appl. Immunohistochem. Mol. Morphol. 17; 102-107.

18. Martín-Villar, E., Scholl, F. G., Gamallo, C., Yurrita, M. M., Muñoz-Guerra, M., Cruces, J. and Quintanilla, M. (2005) Characterization of human PA2.26 antigen (T1alpha-2, podoplanin), a small membrane mucin induced in oral squamous cell carcinomas. Int. J. Cancer. 113; 899-910. 
19. Nagato, T., Yoshida, H., Yoshida, A. and Uehara, Y. (1980) A scanning electron microscope study of myoepithelial cells in exocrine glands. Cell Tissue Res. 209; 1-10.

20. Noda, Y., Amano, I., Hata, M., Kojima, H. and Sawa, Y. (2010) Immunohistochemical examination of the distribution of cells expressed lymphatic endothelial marker podoplanin and LYVE-1 in the mouse tongue tissue. Acta Histochem. Cytochem. 43; 6168 .

21. Ogasawara, S., Kaneko, M. K., Price, J. E. and Kato, Y. (2008) Characterization of anti-podoplanin monoclonal antibodies: critical epitopes for neutralizing the interaction between podoplanin and CLEC-2. Hybridoma (Larchmt) 27; 259-267.

22. Ramirez, M. I., Millien, G., Hinds, A., Cao, Y. X., Seldin, D. C. and Williams, M. C. (2003) Tlalpha, a lung type I cell differentiation gene, is required for normal lung cell proliferation and alveolous formation at birth. Dev. Biol. 256; 61-72.

23. Rishi, A. K., Joyce-Brady, M., Fisher, J., Dobbs, L. G., Floros, J., VanderSpek, J., Brody, J. S. and Williams, M. C. (1995) Cloning, characterization, and development expression of a rat lung alveolar type I cell gene in embryonic endodermal and neural derivatives. Dev. Biol. 167; 294-306.

24. Sawa, Y., Tsuruga, E., Iwasawa, K., Ishikawa, H. and Yoshida, S. (2008) Leukocyte adhesion molecule and chemokine production through lipoteichoic acid recognition by toll-like receptor 2 in cultured human lymphatic endothelium. Cell Tissue Res. 333; 237-252.

25. Sawa, Y. and Tsuruga, E. (2008) The expression of E-selectin and chemokines in the cultured human lymphatic endothelium with lipopolysaccharides. J. Anat. 212; 654-663.

26. Sawa, Y., Ueki, T., Hata, M., Iwasawa, K., Tsuruga, E., Kojima, H., Ishikawa, H. and Yoshida, S. (2008) LPS-induced IL-6, IL-8, VCAM-1, and ICAM-1 expression in human lymphatic endothelium. J. Histochem. Cytochem. 56; 97-109.

27. Schacht, V., Ramirez, M. I., Hong, Y. K., Hirakawa, S., Feng, D., Harvey, N., Williams, M., Dvorak, A. M., Dvorak, H. F., Oliver, G. and Detmar, M. (2003) T1 $\alpha /$ podoplanin deficiency disrupts normal lymphatic vasculature formation and causes lymphedema. EMBO J. 22; 3546-3556.

28. Schacht, V., Dadras, S. S., Johnson, L. A., Jackson, D. G., Hong, Y. K. and Detmar, M. (2005) Up-regulation of the lymphatic marker podoplanin, a mucin-type transmembrane glycoprotein, in human squamous cell carcinomas and germ cell tumors. Am. J. Pathol. 166; 913-921.
29. Sugimoto, Y., Watanabe, M., Oh-hara, T., Sato, S., Isoe, T. and Tsuruo, T. (1991) Suppression of experimental lung colonization of a metastatic variant of murine colon adenocarcinoma 26 by a monoclonal antibody $8 \mathrm{~F} 11$ inhibiting tumor cell-induced platelet aggregation. Cancer Res. 51; 921-925.

30. Suzuki-Inoue, K., Kato, Y., Inoue, O., Kaneko, M. K., Mishima, K., Yatomi, Y., Yamazaki, Y., Narimatsu, H. and Ozaki, Y. (2007) Involvement of the snake toxin receptor CLEC-2, in podoplaninmediated platelet activation, by cancer cells. J. Biol. Chem. 282; 25993-26001.

31. Watanabe, M., Okochi, E., Sugimoto, Y. and Tsuruo, T. (1988) Identification of a platelet-aggregating factor of murine colon adenocarcinoma 26: $\mathrm{Mr} 44,000$ membrane protein as determined by monoclonal antibodies. Cancer Res. 48; 6411-6416.

32. Watanabe, M., Sugimoto, Y. and Tsuruo, T. (1990) Expression of a Mr 41,000 glycoprotein associated with thrombin-independent platelet aggregation in high metastatic variants of murine B16 melanoma. Cancer Res. 50; 6657-6662.

33. Wetterwald, A., Hoffstetter, W., Cecchini, M. G., Lanske, B., Wagner, C., Fleisch, H. and Atkinson, M. (1996) Characterization and cloning of the E11 antigen, a marker expressed by rat osteoblasts and osteocytes. Bone 18; 125-132.

34. Wicki, A., Lehembre, F., Wick, N., Hantusch, B., Kerjaschki, D, and Christofori, G. (2006) Tumor invasion in the absence of epithelial-mesenchymal transition: podoplanin-mediated remodeling of the actin cytoskeleton. Cancer Cell 9; 261-272.

35. Williams, M. C., Cao, Y., Hinds, A., Rishi, A. K. and Wetterwald, A. (1996) Tlalpha protein is developmentally regulated and expressed by alveolar type I cells, choroid plexus and ciliary epithelia of adult rats. Am. J. Respir. Cell Mol. Biol. 14; 577-585.

36. Yu, H., Gibson, J. A., Pinkus, G. S. and Hornick, J. L. (2007) Podoplanin (D2-40) is a novel marker for follicular dendritic cell tumors. Am. J. Clin. Pathol. 128; 776-782.

37. Yuan, P., Temam, S., El-Naggar, A., Zhou, X., Liu, D. D., Lee, J. J. and Mao, L. (2006) Overexpression of podoplanin in oral cancer and its association with poor clinical outcome. Cancer $107 ; 563-569$.

This is an open access article distributed under the Creative Commons Attribution License, which permits unrestricted use, distribution, and reproduction in any medium, provided the original work is properly cited. 